\title{
Efficacy of Pars Plana Vitrectomy with Internal Limiting Membrane Peeling for Treatment of Large Idiopathic Full-Thickness Macular Holes
}

This article was published in the following Dove Press journal: Clinical Ophthalmology

\author{
Suthasinee Sinawat $\mathbb{D}^{\prime}$ \\ Sukita Jumpawong' \\ Tanapat Ratanapakorn' \\ Chavakij Bhoomibunchoo' \\ Yosanan Yospaiboon (D) \\ Supat Sinawat ${ }^{2}$ \\ 'KKU Eye Center, Department of \\ Ophthalmology, Faculty of Medicine, \\ Khon Kaen University, Khon Kaen, \\ Thailand; ${ }^{2}$ Department of Physiology, \\ Faculty of Medicine, Khon Kaen \\ University, Khon Kaen, Thailand
}

Purpose: To evaluate anatomical and visual outcomes of pars plana vitrectomy (PPV) with internal limiting membrane peeling (ILMP) in large idiopathic full-thickness macular holes (FTMH). Predictive factors also formed part of the study.

Patients and Methods: A retrospective review of medical charts and optical coherence tomography images of patients with large idiopathic FTMH $(\geq 400 \mu \mathrm{m})$ was conducted.

Results: One hundred and fifty-eight eyes of 155 patients with a mean age of $62.94 \pm 7.50$ years were included in the study. Mean preoperative visual acuity (VA) was $1.26 \pm 0.36$ $\log$ MAR. Mean preoperative minimum linear diameter (MLD) and basal linear diameter (BLD) were $644.89 \pm 136.85 \mu \mathrm{m}$ and $1208.11 \pm 307.14 \mu \mathrm{m}$, respectively. At 12 weeks postoperative follow-up, FTMH closure rate was $61.39 \%$ and mean postoperative BCVA was $0.92 \pm 0.36 \log$ MAR. Multivariate logistic regression analyses showed the anatomical outcome was significantly associated with $\mathrm{BLD} \leq 1200 \mu \mathrm{m}$, preoperative vitreomacular interface (VMI) disorder and extended ILMP (a radius of 2 disc diameters), whereas only postoperative ellipsoid loss $\leq 500 \mu \mathrm{m}$ was significantly associated with visual outcome. In subgroup analyses, the large FTMH group $(400-600 \mu \mathrm{m})$ had significantly better results in closure rate $(70.97 \%$ vs $55.21 \%, P=0.047)$ and visual results $(P=0.031)$ than the extralarge FTMH group $(>600 \mu \mathrm{m})$.

Conclusion: PPV with conventional ILMP provided relatively low closure rate in large FTMH. Surgical success was significantly associated with BLD $\leq 1200 \mu \mathrm{m}$, no preoperative VMI abnormality, extended ILMP and postoperative ellipsoid defect $\leq 500 \mu \mathrm{m}$. Therefore, we suggest the extended ILMP if conventional ILMP technique is used. The large FTMH group had significantly better surgical outcomes than the extra-large group, so there is a need for reclassification of large FTMH.

Keywords: full-thickness macular hole, internal limiting membrane peeling, conventional, complete flap, large macular hole

\section{Introduction}

Macular hole is one of the major causes of central visual loss worldwide. The incidence for idiopathic macular hole is 7.8 per 100,000 population/year. ${ }^{1}$ The tractional force in a vitreofoveal surface on a macular area can cause a disruption of the retinal pigment epithelium (RPE) layer, resulting in a full-thickness macular hole (FTMH).

Pars plana vitrectomy (PPV), combined with internal limiting membrane peeling (ILMP), and intravitreal gas tamponade is the standard treatment for FTMH. ${ }^{2}$ For FTMH $>400 \mu \mathrm{m}$, a recent review found a significant benefit of face-down posturing
Correspondence: Suthasinee Sinawat; Yosanan Yospaiboon

KKU Eye Center, Department of Ophthalmology, Faculty of Medicine, Khon Kaen University, I 23 Mittraparb Highway, Khon Kaen, 40002, Thailand Tel +66 43348383

Email ssuthasinee@kku.ac.th; yyosanan@gmail.com 
on successful hole closure. ${ }^{3}$ ILMP has been shown to increase closure rate and prevent reopening, whereas a gas tamponade with face-down positioning keeps the macula dry and allows glial cell migration to bridge the gap between the retinal edges. ${ }^{4,5}$ A longer period of intravitreal gas tamponade such as perfluoropropane $\left(\mathrm{C}_{3} \mathrm{~F}_{8}\right)$ has also been proven to increase treatment success especially in large $(>400 \mu \mathrm{m})$ and chronic ( $>1$ year) FTMH. ${ }^{6}$ Previous studies reported the FTMH closure rate was quite high $(85-100 \%){ }^{7}$ however, the main negative predictive factors included large hole size and chronic macular hole. From previous publications, few studies evaluate the surgical success rate of conventional ILMP and the impact factors of an idiopathic large FTMH specifically. $^{8-11}$

The purpose of this study is to determine an anatomical hole closure and a visual outcome after surgical treatment of large idiopathic FTMH, and also evaluate the predictive factors and the possible adverse events which are caused by a disease progression or related to treatment.

\section{Patients and Methods}

In the study, we reviewed the medical records and macular images of patients with idiopathic large FTMH who had undergone pars plana vitrectomy (PPV) at KKU Eye Center, Srinagarind hospital, Khon Kaen University, Khon Kaen, Thailand between January 2008 and December 2017. The macula was evaluated using a spectral-domain optical coherence tomography (SD-OCT) device (Spectralis, Heidelberg Engineering, Heidelberg, Germany). The minimum linear diameter (MLD) was defined as FTMH size when the horizontal SD-OCT passed through the center of the macular hole. Following the International Vitreomacular Traction Study (IVTS) classification, the definition of large FTMH in our study was FTMH with MLD $>400 \mu \mathrm{m} .{ }^{13}$ We also measured basal linear diameter
(BLD) and calculated the macular hole closure index (MHCI). The MHCI was calculated as the sum length of the detached photoreceptor arms divided by basal linear diameter (Figure 1). ${ }^{14}$ The detached photoreceptor arm is defined as a straight line from the broken end point of the external limiting membrane (ELM) to the junction of the detached photoreceptors with RPE. The disc-to-fovea distance (DFD) or retinal displacement is measured from the center of fovea to a landmark at the temporal disc margin. The foveal center of FTMH is guided by OCT; the end of half-length of BLD was the center of fovea. All lengths were measured by two independent investigators (SS, SJ) using the built-in caliper of the OCT software and the average results were used for the analyses. The postoperative dissociated optic nerve fiber layer (DONFL) appearance was observed and documented. Swelling of the arcuate retinal nerve fiber layer (SANFL) or DONFL manifested as small dimpling of the disaggregated nerve fiber layer on SD-OCT and dark dots or lines on the red free image. Inclusion criteria included (1) age $>50$ years (2) FTMH with a minimum linear diameter (MLD) $>400$ $\mu \mathrm{m}$ confirmed by spectral-domain optical coherent tomography (SD-OCT; Spectralis, Heidelberg Engineering, Heidelberg, Germany), and (3) three months of follow-up. Exclusion criteria included (1) refractive error $\geq 3$ diopters (2) glaucoma (3) macular scarring (4) media opacity resulting in poor OCT image quality (5) macular hole-induced retinal detachment (6) history of traumatic eye or head injury (7) previous vitreoretinal disorder, and (8) previous vitreoretinal surgery.

The study protocol had obtained approval of the institutional review board and Khon Kaen University Ethics Committees in Human Research (IRB no. 00001189). The procedures conformed to the tenets of the Declaration of Helsinki. Patient consents to review their medical records

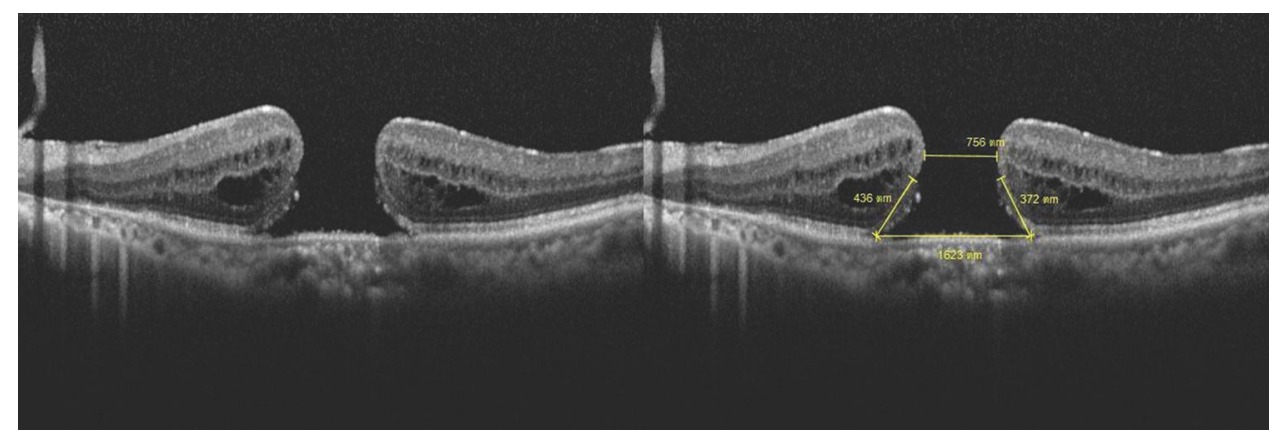

Figure I The macular hole closure index $(\mathrm{MCHI})$ was calculated as the sum length of the detached photoreceptor arms divided by basal linear diameter. Therefore, $\mathrm{MHCl}$ of the example was calculated as $(436+37 I) / 1623$ which equals to 0.50 . 
were not required due to the retrospective study design, but the data in case report forms had no linkage to the patient identities and the researcher respected the confidentiality of the patient data. The Invitation Research Fund had no role in this study.

The surgery consisted of sutureless 3-port 23/25-gauge, posterior hyaloid separation and ILMP in all cases. The posterior vitreous disengagement was performed with microincision instruments or a silicone-tipped aspiration cannula. Forceps were used to peel ILM $360^{\circ}$ around the macular hole. In conventional ILMP, the ILM was completely removed from the edge of the macular hole. In invertedflap ILMP, the ILM was peeled toward the macular hole and retained at the edge of the hole. A non-expansile mixture of $20 \%$ sulfur hexafluoride (SF6) or $14 \%$ perfluoropropane $(\mathrm{C} 3 \mathrm{~F} 8)$ was injected at the end of the procedure. Patients were instructed to remain in a facedown position for 1-2 weeks depending on the surgical outcomes at one week postoperatively. If the FTMH was not closed, the patient was asked to maintain the posture for another week.

The primary outcome observed was the anatomical closure rate. According to the previous study, the macular hole closure was classified at three months after treatment into four types as shown in Figure 2. Regular foveal contour was classified as U-type. Closed hole with deep foveal contour was classified as V-type and a retinal pigment epithelium defect might be visible under the fovea. Closed hole with irregular foveal contour classification was classified as irregular or W-type. Unclosed macular hole with flattened cuff of retinal edema around the hole was classified as Open type and defined as a failure. ${ }^{15}$ The secondary outcomes were the means of best-corrected visual acuity (BCVA), predictive factors and related adverse events. All these outcomes were collected at baseline, 1, 4 and 12 weeks after the surgical treatment. SD-OCT images were reviewed for $\mathrm{MHCI}$, anatomical closure, closure type, extension of ellipsoid layer loss and foveolar thickness.

All statistical analyses were performed with STATA version 10.0 (StataCorp, College Station, TX, USA). Mean \pm standard deviation (SD) used to describe the continuous variables, whereas frequency and percentage were used in categorical variables. The BCVA was converted to logarithm of the minimal angle of resolution ( $\log$ MAR) for facilitating statistical analyses. Pearson's chi-square tests and Fisher's exact tests were performed to determine the significant differences between categorical variables. Univariate and multivariate logistic regression was used to analyze the significant correlations between independent variables and the outcomes. P-value $<0.05$ is considered for statistical significance.

\section{Results}

One hundred and fifty-eight eyes of 155 patients were included in the study; 113 were female and 42 were male. Three patients underwent surgery for bilateral large FTMH. The mean age was $62.94 \pm 7.50$ years. One hundred and ten eyes $(69.62 \%)$ had blurred vision for more than 6 months. Mean duration of symptoms was $12.63 \pm 11.04$ months, with 6 years as the longest duration period. Twenty-seven patients had diabetes mellitus as their medical condition. Forty-nine eyes had preoperative vitreomacular interface (VMI)

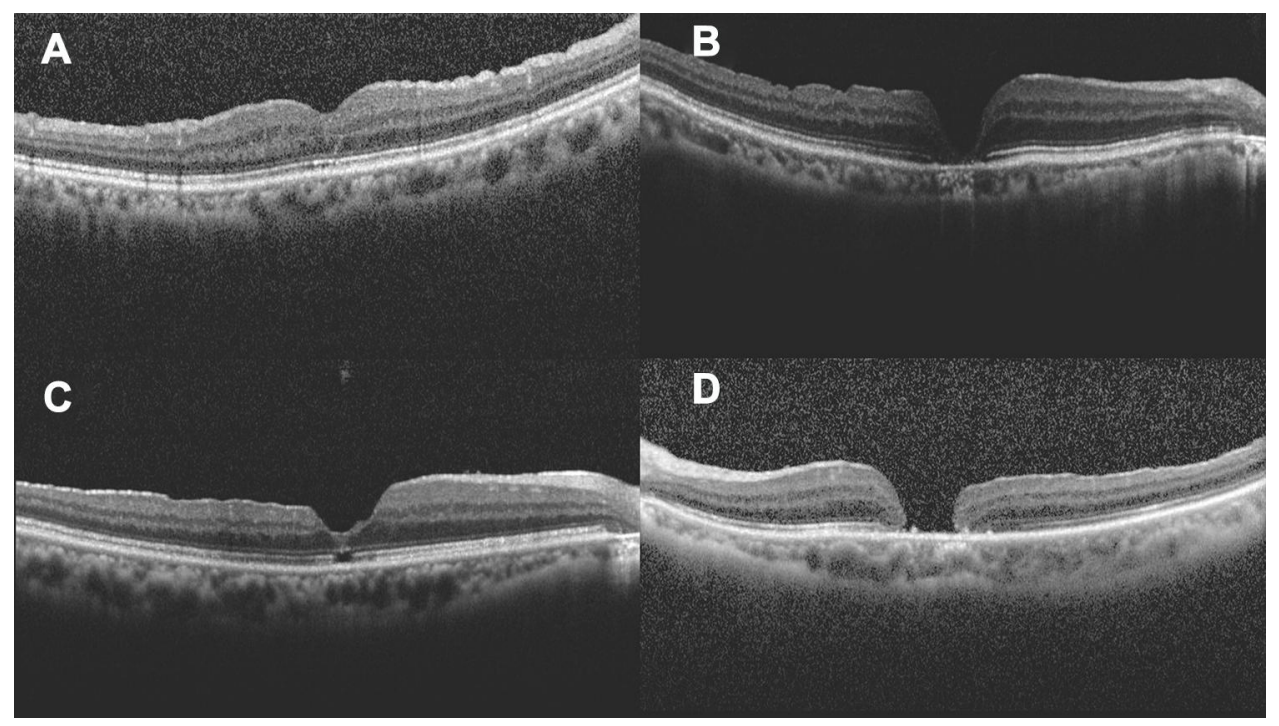

Figure 2 Classification of macular hole closure based on OCT images: (A) U-type, (B) V-type, (C) irregular or W-type, (D) open-type or unclosed hole. 
abnormalities: epimacular membrane (EMM) in 32 eyes, vitreomacular traction (VMT) in 13 eyes, vitreomacular adhesion (VMA) in 1 eye, foveoschisis in 1 eye, whereas both EMM and VMT were found in two eyes. Mean preoperative MLD and BLD were $644.89 \pm 136.85 \mu \mathrm{m}$ and $1208.11 \pm 307.14 \mu \mathrm{m}$, respectively. The majority of patients (86.71\%) had $\mathrm{MHCI} \leq 0.5$, mean preoperative MHCI was $0.45 \pm 0.13$. Mean baseline $\log$ MAR BCVA was $1.25 \pm 0.36$. Peripheral retinal abnormalities were recorded in 2 eyes including lattice retinal degeneration ( 1 eye) and retinal break (1 eye). Most cases (83.54\%) were phakic; however, phacovitrectomy was conducted in 55 eyes (41.67\%). Conventional ILMP was performed in almost all cases, 152 eyes (96.20\%). Ninety-eight eyes (64.47\%) and 54 eyes $(35.53 \%)$ underwent conventional ILMP with a radius of 2 discs diameters (DD) and 1 DD, respectively. There were only 6 eyes that underwent inverted-flap ILMP. The majority of cases $(77.22 \%)$ used sulfur hexafluoride $\left(\mathrm{SF}_{6}\right)$ as gas tamponade. Silicone oil was used in one patient who also had an inferior retinal break. Patients' demographic data, clinical characteristics and surgical outcomes are presented in Table 1.

Anatomical closures were demonstrated in 97 eyes (61.39\%) at 12-week follow-up period. The mean postoperative $\log$ MAR BCVA was $0.92 \pm 0.36$ at 12-week follow-up period. The majority of cases with macular hole closure (85.57\%) had visual improvement; mean final BCVA improvement was $0.32 \pm 0.97 \log$ MAR (approximately 2 Snellen lines improvement). A subgroup analysis was performed; 61 eyes (38.61\%) were categorized as large FTMH $(400-600 \mu \mathrm{m})$ and 97 eyes $(61.49 \%)$ were classified as extra-large FTMH $(>600 \mu \mathrm{m})$. There was no statistically significant difference in demographic data, preoperative BCVA, lens status and preoperative VMI disorder between the large FTMH group and extra-large FTMH groups. According to the larger macular hole size, the extra-large FTMH group had significantly lower MHCI. Considering the results, the large FTMH group had statistically significantly better surgical outcome. The closure rate was significantly higher in the large FTMH group $(70.97 \%)$ than in the

Table I Demographic Data, Clinical Characteristics and Surgical Outcomes

\begin{tabular}{|c|c|c|c|c|}
\hline & Total & $\begin{array}{l}\text { Large FTMH } \\
(400-600 \mu \mathrm{m}) \mathrm{N}=61\end{array}$ & $\begin{array}{l}\text { Extra-Large FTMH } \\
(>600 \mu \mathrm{m}) \mathrm{N}=97\end{array}$ & $P$ value \\
\hline \multicolumn{5}{|l|}{ Baseline demographics } \\
\hline - Age, years $\pm S D$ & $62.94 \pm 7.50$ & $62.36 \pm 5.80$ & $63.32 \pm 8.43$ & 0.439 \\
\hline - Gender, female: male & 113: 42 & 40: 21 & 73: 21 & 0.098 \\
\hline - Diabetes mellitus, n (\%) & $28(17.72)$ & $8(12.90)$ & $20(20.83)$ & 0.202 \\
\hline - Duration, months $\pm S D$ & $|2.63 \pm| 1.04$ & $10.65 \pm 6.75$ & $13.91 \pm 12.96$ & 0.069 \\
\hline \multicolumn{5}{|l|}{ Clinical characteristics } \\
\hline - Preoperative BCVA, logMAR \pm SD & $1.26 \pm 0.36$ & $1.25 \pm 0.39$ & $1.36 \pm 0.44$ & 0.087 \\
\hline - Lens status, n Phakic: Pseudophakic & 132: 26 & 51: 11 & $81: 15$ & 0.726 \\
\hline - Minimum linear diameter, $\mu \mathrm{m} \pm \mathrm{SD}$ (range) & $644.89 \pm 136.85(404-1069)$ & $513.85 \pm 52.24(404-600)$ & $729.53 \pm 103.70(607-1069)$ & $<0.001$ \\
\hline - Basal linear diameter, $\mu \mathrm{m} \pm \mathrm{SD}$ (range) & $1208.1 \mid \pm 307.14$ (667-3662) & III $7.65 \pm 409.4 \mid(667-3662)$ & $\mid 266.54 \pm 198.72(867-2 \mid 86)$ & 0.003 \\
\hline - Macular hole closure index, mean \pm SD & $0.45 \pm 0.13$ & $0.52 \pm 0.13$ & $0.41 \pm 0.12$ & $<0.001$ \\
\hline - Preoperative VMI disorder, n (\%) & $46(29.11)$ & $18(29.03)$ & $28(29.17)$ & 0.986 \\
\hline \multicolumn{5}{|l|}{ Surgical procedure } \\
\hline - Extended ILMP, n (\%) & $98(62.03)$ & $38(61.29)$ & $60(62.50)$ & 0.878 \\
\hline - Type of tamponade, $\mathrm{nSF}_{6}: \mathrm{C}_{3} \mathrm{~F}_{8}$ & 122: 35 & 50: 12 & 72: 23 & 0.475 \\
\hline - Phacovitrectomy, n (\%) & $55(4 I .67)$ & $16(31.37)$ & $38(46.91)$ & 0.077 \\
\hline \multicolumn{5}{|l|}{ Postoperative 12 weeks } \\
\hline - Postoperative BCVA, logMAR \pm SD & $0.99 \pm 0.48$ & $0.89 \pm 0.36$ & $1.06 \pm 0.54$ & 0.031 \\
\hline - Closure rate, n (\%) & $97(61.39 \%)$ & $44(70.97 \%)$ & $53(55.21 \%)$ & 0.047 \\
\hline - U: V: W closure type, $n$ & 34: 45: 18 & 20: 15: 9 & 14: 30: 9 & 0.072 \\
\hline - Foveolar thickness, $\mu \mathrm{m} \pm \mathrm{SD}$ & $144.77 \pm 57.10$ & $166.07 \pm 50.66$ & $127.09 \pm 56.52$ & 0.001 \\
\hline - Ellipsoid loss, $\mu \mathrm{m} \pm$ SD & $478.02 \pm 438.29$ & $254.09 \pm 248.01$ & $663.92 \pm 475.53$ & $<0.001$ \\
\hline - Change of DFD, $\mu \mathrm{m} \pm \mathrm{SD}$ & $134.60 \pm 127.30$ & $150.19 \pm 156.42$ & $124.53 \pm 103.97$ & 0.217 \\
\hline - Presence of DONFL, n (\%) & $116(73.42)$ & $49(79.03)$ & $67(69.79)$ & 0.199 \\
\hline
\end{tabular}

Abbreviations: FTMH, full-thickness macular hole; BCVA, best corrected visual acuity; logMAR, logarithm of the minimum angle of resolution; VMI, vitreomacular interface; ILMP, internal limiting membrane peeling; $\mathrm{SF}_{6}$, sulfur hexafluoride; $\mathrm{C}_{3} \mathrm{~F}_{8}$, perfluoropropane; DFD, disc-to-fovea distance; DONFL, dissociated optic nerve fiber layer. 
extra-large FTMH group (55.21\%), $P=0.047$. Furthermore, the patients with hole closure in the large FTMH group also had significantly more foveolar thickness $(P=0.001)$ and shorter length of ellipsoid loss $(\mathrm{P}<0.001)$. Regarding the visual outcome at 12-week follow-up period, mean postoperative $\log$ MAR BCVA were $0.89 \pm 0.36$ in the large FTMH group and $1.06 \pm 0.54$ in the extra-large FTMH group, meaning the visual result was significantly better in the large FTMH group $(P=0.031)$ (Table 1).

Univariate and multivariate logistic regression analyses performed to examine the associated factors of surgical outcomes. The following factors affected anatomical outcomes on univariate analysis: MLD $\leq 600 \mu \mathrm{m}, \mathrm{BLD} \leq 1200$ $\mu \mathrm{m}$ and extended ILMP. Stepwise multivariate analysis demonstrated that BLD $\leq 1200 \mu \mathrm{m}$, preoperative VMI abnormality and extended ILMP were significantly associated with macular hole closure (Table 2). Univariate analysis found multiple variables associated with visual outcome including preoperative BCVA, perfluoropropane gas tamponade, U-type closure, foveolar thickness $>150$ $\mu \mathrm{m}$ and length of ellipsoid loss $\leq 500 \mu \mathrm{m}$; however, multivariate analysis revealed only the length of ellipsoid loss $\leq 500 \mu \mathrm{m}$ was significantly associated with the visual outcome (Table 3).
The most common complication was a secondary cataract $(24.68 \%)$. Other complications included epimacular membrane (4 eyes), retinal trauma (4 eyes), widening macular atrophy (1 eye), retrobulbar hemorrhage with globe perforation ( 1 eye), an iatrogenic retinal break (1 eye) and rhegmatogenous retinal detachment (1 eye). In cases of successful anatomical closure, no FTMH reopening was detected during the follow-up period.

\section{Discussion}

In previous publications of large macular holes, there were variations in the definition and in macular holes measurement methods. In the IVTS study, large FTMH has a narrowest width slightly more than $400 \mu \mathrm{m} .{ }^{13}$ Our retrospective study demonstrates that the anatomical closure of large FTMH with MLD $>400 \mu \mathrm{m}$ is $61.39 \%$ after combined PPV with conventional ILMP and gas tamponade, which is comparable with previous studies. ${ }^{16,17}$ Ip et al. reported $56 \%$ anatomical closure in large FTMH with MLD $\geq 400 \mu \mathrm{m}$, but no ILMP was operated in this prospective case series. ${ }^{16}$ Susini and Gastaud also reported only $50 \%$ of closure in FTMH $>500 \mu \mathrm{m} .{ }^{17}$ The recent studies reported quite high closure rates (77.8-92.40\%) in large macular holes after PPV with conventional ILMP. $^{8-12}$ Narayanan et al. used the BLD as hole size while

Table 2 Univariate and Multivariate Analysis of Factors Associated with Macular Hole Closure

\begin{tabular}{|c|c|c|c|c|c|c|}
\hline & \multicolumn{3}{|c|}{ Univariate Analysis } & \multicolumn{3}{|c|}{ Multivariate Analysis } \\
\hline & Crude OR & $95 \% \mathrm{Cl}$ & $P$-value & $\begin{array}{l}\text { Adjusted } \\
\text { OR }\end{array}$ & $95 \% \mathrm{Cl}$ & $P$-value \\
\hline \multicolumn{7}{|l|}{ Baseline } \\
\hline Age $\leq 60$ years & 1.90 & $0.92,4.01$ & 0.063 & 1.82 & $0.87,3.81$ & 0.110 \\
\hline Male & 0.73 & $0.34,1.58$ & 0.379 & & & \\
\hline Diabetes mellitus & 0.47 & $0.19,1.18$ & 0.073 & 0.47 & $0.19,1.17$ & 0.106 \\
\hline Duration $\leq 12$ months & 1.32 & $0.64,2.70$ & 0.416 & & & \\
\hline Preoperative $\mathrm{BCVA} \leq \mathrm{I} \log \mathrm{MAR}$ & 1.20 & $0.60,2.4 \mathrm{I}$ & 0.589 & & & \\
\hline Minimum linear diameter $\leq 600 \mu \mathrm{m}$ & 1.98 & $0.96,4.18$ & 0.047 & 1.24 & $0.54,2.84$ & 0.608 \\
\hline Basal linear diameter $\leq \mathrm{I} 200 \mu \mathrm{m}$ & 2.83 & $1.38,5.84$ & 0.002 & 2.93 & $1.37,6.29$ & 0.006 \\
\hline Macular hole closure index $\leq 0.5$ & 0.53 & $0.24,1.12$ & 0.073 & 0.70 & $0.31,1.58$ & 0.384 \\
\hline Preoperative VMI disorder & 1.90 & $0.86,4.37$ & 0.087 & 2.46 & $1.06,5.70$ & 0.036 \\
\hline \multicolumn{7}{|l|}{ Surgical procedure } \\
\hline Extended ILMP & 0.46 & $0.23,0.95$ & 0.021 & 0.48 & $0.23,0.98$ & 0.044 \\
\hline Perfluoropropane $\left(\mathrm{C}_{3} \mathrm{~F}_{8}\right)$ tamponade & 1.46 & $0.62,3.62$ & 0.349 & & & \\
\hline Phacovitrectomy & 0.86 & $0.40,1.87$ & 0.680 & & & \\
\hline \multicolumn{7}{|l|}{ Postoperative I 2 weeks } \\
\hline Change of DFD $\leq 200 \mu \mathrm{m}$ & 1.34 & $0.57,3.09$ & 0.456 & & & \\
\hline Presence of DONFL & 1.46 & $0.66,3.16$ & 0.303 & & & \\
\hline
\end{tabular}

Abbreviations: OR, odds ratio; $\mathrm{Cl}$, confidence interval; BCVA, best corrected visual acuity; logMAR, logarithm of the minimum angle of resolution; $\mathrm{VMI}$, vitreomacular interface; ILMP, internal limiting membrane peeling; DFD, disc-to-fovea distance; DONFL, dissociated optic nerve fiber layer. 
Table 3 Univariate and Multivariate Analysis of Factors Associated Postoperative Visual Outcome

\begin{tabular}{|c|c|c|c|c|c|c|}
\hline & \multicolumn{3}{|c|}{ Univariate Analysis } & \multicolumn{3}{|c|}{ Multivariate Analysis } \\
\hline & Mean Difference & $95 \% \mathrm{Cl}$ & P-value & $\begin{array}{l}\text { Adjusted Mean } \\
\text { Difference }\end{array}$ & $95 \% \mathrm{Cl}$ & P-value \\
\hline \multicolumn{7}{|l|}{ Baseline } \\
\hline Age $\leq 60$ years & -0.10 & $-0.30,0.09$ & 0.290 & & & \\
\hline Male & -0.16 & $-0.38,0.06$ & 0.156 & & & \\
\hline Diabetes mellitus & -0.07 & $-0.36,0.21$ & 0.615 & & & \\
\hline Duration $\leq 12$ months & -0.01 & $-0.21,0.20$ & 0.956 & & & \\
\hline Preoperative $B C V A \leq I \log M A R$ & -0.23 & $-0.42,-0.04$ & 0.019 & -0.15 & $-0.33,0.03$ & 0.110 \\
\hline Minimum linear diameter $\leq 600 \mu \mathrm{m}$ & -0.15 & $-0.34,0.04$ & 0.130 & 0.03 & $-0.17,0.23$ & $0.77 \mathrm{I}$ \\
\hline Basal linear diameter $\leq \mathrm{I} 200 \mu \mathrm{m}$ & -0.07 & $-0.27,0.12$ & 0.461 & & & \\
\hline Macular hole closure index $\leq 0.5$ & 0.10 & $-0.09,0.30$ & 0.303 & & & \\
\hline Preoperative VMI disorder & 0.03 & $-0.17,0.24$ & 0.757 & & & \\
\hline \multicolumn{7}{|l|}{ Surgical procedure } \\
\hline Extended ILMP & -0.16 & $-0.37,0.05$ & 0.134 & -0.07 & $-0.27,0.13$ & 0.489 \\
\hline Perfluoropropane $\left(\mathrm{C}_{3} \mathrm{~F}_{8}\right)$ tamponade & 0.23 & $0.00,0.45$ & 0.046 & 0.21 & $-0.00,0.42$ & 0.055 \\
\hline Phacovitrectomy & 0.02 & $-0.18,0.23$ & 0.843 & & & \\
\hline \multicolumn{7}{|l|}{ Postoperative 12 weeks } \\
\hline U-type closure & -0.26 & $-0.46,-0.07$ & 0.009 & -0.14 & $-0.38,0.11$ & 0.263 \\
\hline Foveolar thickness $>150 \mu \mathrm{m}$ & 0.23 & $0.04,0.42$ & 0.016 & 0.03 & $-0.2 \mathrm{I}, 0.27$ & 0.830 \\
\hline Length of ellipsoid loss $\leq 500 \mu \mathrm{m}$ & 0.39 & $0.20,0.58$ & $<0.001$ & 0.30 & $0.07,0.52$ & 0.011 \\
\hline Change of DFD $\leq 200 \mu \mathrm{m}$ & 0.14 & $-0.10,0.64$ & 0.250 & & & \\
\hline Presence of DONFL & -0.10 & $-0.33,0.13$ & 0.398 & & & \\
\hline
\end{tabular}

Abbreviations: OR, odds ratio; $\mathrm{Cl}$, confidence interval; $\mathrm{BCVA}$, best corrected visual acuity; logMAR, logarithm of the minimum angle of resolution; VMI, vitreomacular interface; ILMP, internal limiting membrane peeling; DFD, disc-to-fovea distance; DONFL, dissociated optic nerve fiber layer.

MLD was $243-840 \mu \mathrm{m}$ in range. ${ }^{8}$ Velez-Montoya et al. recruited only 12 patients with MLD $>400 \mu \mathrm{m} .{ }^{9}$ Yamashita et al. did not give information of hole duration in their study, which is a significant prognostic factor for hole closures. The number of patients with stage 3 FTMH in their study was much higher than found in our study ( $58.50 \%$ vs $9.49 \%)$, this may refer to more chronicity of our patients. ${ }^{10}$ The previous studies have reported that an anatomical outcome was limited in patients who developed FTMH for more than a year. ${ }^{18,19}$ Our study also recruited FTMH patients with larger both mean MLD and BLD, so these may be one of the reasons for a lower success rate.

The majority of our cases used dual dye staining. We did not use indocyanine green (ICG) mainly because of its potential toxicity, furthermore, ICG-assisted ILMP may provide a lower success rate. ${ }^{20}$ Three techniques of internal limiting membrane peeling have been used for treatment of FTMH including conventional ILMP, inverted-flap and free-flap technique. Although a prospective multicenter randomized controlled trial of large FTMH with minimum diameter $>400 \mu \mathrm{m}$ confirms no difference in closure rates between all three ILMP techniques, the sample size is too limited in each group and the inverted-flap technique seems to have induced a faster and more significant visual recovery. ${ }^{9}$ Michalewska et al. proposed the inverted ILMP technique via a prospective RCT. They found that inverted ILMP with an air tamponade could provide both better anatomical and visual outcomes than conventional ILMP in large FTMH. Moreover, an inverted-flap technique prevents the postoperative reopening of macular holes. ${ }^{11}$

A large retrospective comparative study also demonstrated that inverted ILMP was more effective than conventional technique for idiopathic large FTMH and myopic macular holes. ${ }^{12}$ A recent meta-analysis suggested that the inverted-flap technique is more effective in achieving anatomical closure but not visual outcome. ${ }^{21}$ Even though inverted ILMP appeared to be beneficial in large FTMH, unfortunately, there were only 6 cases that underwent inverted-flap ILMP in this retrospective study that recruited a too small sample size to effectively analyze the statistical significance.

The ILMP has proven to improve the closure rate of the macular holes, but can have several consequences on retinal structure. Retinal displacement with temporal thinning and dissociated optic nerve fiber layer (DONFL) appearance could demonstrate on SD-OCT, whereas 
reduction in macular vessel density and smaller foveal avascular zone (FAZ) areas may show on optical coherence tomography angiography (OCTA). ${ }^{22-24}$ We found that the retinal displacement and presence of DONFL did not relate to the surgical outcomes, however, en-face OCT will provide more information than SD-OCT especially the absolute number of dimples and their density. Four patients had deep retinal dimples detected on SD-OCT, these retinal trauma may be the result of too deep grasping of initial ILMP. There is no consensus, regarding the extent of the area of internal limiting membrane that is subject to optimal peeling. ${ }^{25,26}$ Modi et al. demonstrated that there is no significant differences in hole closure rates. However, there were better visual results in 3-mm ILMP group compared to 5-mm ILMP group. ${ }^{27}$ Bae et al. carried out a randomized controlled trial with ILMP radii of 0.75 and 1.5 disc diameter (DD), there were no differences in anatomical closure and visual outcome between groups. ${ }^{18}$ Although Modi et al. and Bae et al. enrolled patients from all stages of FTMH, large FTMH (stage 3 and 4) contributed $38.33 \%$ and $46 \%$ of cases, respectively. ${ }^{18,27}$ Yao et al. evaluated the impact of extended ILMP via a prospective RCT. They found that ILMP with 2 disc diameters (DD) in radii tends to provide higher closure rate and better visual result in FTMH with $\mathrm{MHCI} \leq 0.5 .^{28}$ Our hypothesis suggests that there may be an optimal ILMP area for a set size of FTMH to reduce retinal disturbance and avoid retinal complications. In this retrospective study, univariate and multivariate analyses found that extended ILMP was associated with anatomical closure; therefore, we highly recommend extended ILMP for treatment of large idiopathic FTMH.

The gas tamponade becomes a standard procedure in combination with PPV and ILMP for an anatomical success and a good visual outcome. A recent meta-analysis found both sulfur hexafluoride $\left(\mathrm{SF}_{6}\right)$ and perfluoropropane appear to have achieved similar outcomes and risk of adverse events, but $\mathrm{SF}_{6}$ provide more rapid visual recovery. ${ }^{29}$ In our study, there were no significant difference in the closure rate between $\mathrm{SF}_{6}$ and $\mathrm{C}_{3} \mathrm{~F}_{8}$ tamponade. Although univariate analysis revealed $\mathrm{C}_{3} \mathrm{~F}_{8}$ gas associated with visual outcomes, however, this was not demonstrated in multivariate analysis.

Univariate analysis revealed many factors associated with visual outcomes. Multivariate analysis showed there was only one factor, postoperative lengths of ellipsoid defects $\leq 500 \mu \mathrm{m}$, significantly associated with visual outcome in our study. Our result was in concordance with many previous studies that reported a significant correlation between ellipsoid defects and postoperative visual acuity. $^{30,31}$ Other variables associated with visual outcomes in prior publications were age, sex, preoperative BCVA, macular hole size, choroidal thickness, closure type, and foveolar thickness. ${ }^{10,11,32,33}$ Michalewska et al. reported that a foveal thickness had a positive correlation with final visual acuity, in addition, U-closure type and normal foveal thickness were good prognostic factors for postoperative visual outcome. ${ }^{15}$ Our results, however, found no statistically significant correlation amongst these variables and postoperative BCVA.

The Manchester large macular hole study divided FTMH with MLD $>400 \mu \mathrm{m}$ into quartiles; 400-477 $\mu \mathrm{m}$, $478-558 \mu \mathrm{m}, 559-649 \mu \mathrm{m}$ and $\geq 650 \mu \mathrm{m}$. The closure rate in the largest FTMH group was $76 \%$. They also found that if cutoff was $>630 \mu \mathrm{m}$ of FTMH size, then the closure rate after a PPV with conventional ILMP fell below the current accepted standards of around 90\%. Therefore they suggested that there was a need for a reclassification of large FTMH. ${ }^{34}$ Yamashita et al. report that large FTMH group (400-700 $\mu \mathrm{m}$ ), had statistically significant higher closure rate and better visual results than the super extra-large FTMH $(>700 \mu \mathrm{m})$. However, both conventional ILMP and inverted-flap techniques were used in their study. ${ }^{10}$ We categorized the patients into 2 groups depending on the hole size, large FTMH group (400-600 $\mu \mathrm{m})$ and extralarge FTMH group $(>600 \mu \mathrm{m})$. In subgroup analysis, we also found a statistically significant difference in both anatomical and visual outcomes. This consistency should lead to new criteria of large FTMH in future studies. Intraoperative optical coherence tomography (iOCT) recently provides intraoperative information that could predict the FTMH closure speed, however, a large prospective clinical trial is needed. ${ }^{35}$

The strength of our study was to evaluate specifically the anatomical, visual outcomes and predictive factors in a large series of patients with large idiopathic FTMH. Even thoughconventional ILMP was mostly used in our study, tmore than half of the patients underwent the extended ILMP technique, which was statistically significantly associated with anatomical closure. Although the study design is retrospective with a relatively shortterm follow-up, we are confident that there are no missing data in the reviews of medical charts and OCT images. The collected data are sufficient for the statistical analyses. 


\section{Conclusions}

PPV combined with conventional ILMP and gas tamponade for large idiopathic FTMH had a relatively low closure rate. The surgical outcomes were significantly associated with BLD $\leq 1200 \mu \mathrm{m}$, preoperative VMI abnormality, extended ILMP and postoperative ellipsoid defect $\leq 500 \mu \mathrm{m}$. Therefore, we suggest the extended ILMP if the conventional ILMP technique is used; however, the combined surgical techniques with or without adjunctive substances should be further studied using advance noninvasive retinal imaging technology with the aim to improve treatment success, especially the visual outcomes. The large FTMH group had significantly better surgical outcomes than the extra-large FTMH group, so the reclassification of large FTMH may be needed in future studies.

\section{Acknowledgments}

The authors would like to thank Dr. Watcharaporn Thongmee for illustrating the figures and Dr. Kaewjai Thepsuthammarat, Clinical Epidemiology Unit, Faculty of Medicine, Khon Kaen University for helping in statistical analyses. We would like to acknowledge Mr. Gurdeep Singh for the English language editing of this manuscript via the KKU Publication Clinic (Thailand).

\section{Disclosure}

The authors declare no conflicts of interest in this work.

\section{References}

1. McCannel CA, Ensminger JL, Diehl NN, et al. Population-based incidence of macular holes. Ophthalmology. 2009;116:1366-1369. doi:10.1016/j.ophtha.2009.01.052

2. Parravano M, Giansanti F, Eandi CM, et al. Vitrectomy for idiopathic macular hole. Cochrane Database Syst Rev. 2015;5:CD009080.

3. Xia S, Zhao XY, Wang EQ, et al. Comparison of face-down posturing with nonsupine posturing after macular hole surgery: a meta-analysis. BMC Ophthalmol. 2019;19:34. doi:10.1186/s12886-019-1047-8

4. Wollensak G, Spoerl E, Grosse G, et al. Biomechanical significance of the human internal limiting lamina. Retina. 2006;26:965-968. doi:10.1097/01.iae.0000250001.45661.95

5. Madreperla SA, Geiger GL, Funata M, et al. Clinicopathologic correlation of a macular hole treated by cortical vitreous peeling and gas tamponade. Opthalmology. 1994;101:682-686. doi:10.1016/S01616420(94)31278-4

6. Madi HA, Masri I, Steel DH. Optimal management of idiopathic macular holes. Clin Ophthalmol. 2016;10:97-116. doi:10.2147/ OPTH.S96090

7. Steel DH, Lotery AJ. Idiopathic vitreomacular traction and macular hole: a comprehensive review of pathophysiology, diagnosis, and treatment. Eye. 2013;27(Suppl 1):S1-21. doi:10.1038/eye.2013.212

8. Narayanan R, Singh SR, Taylor S, et al. Surgical outcomes after inverted internal limiting membrane flap versus conventional peeling for very large macular holes. Retina. 2018:1-5.
9. Velez-Montoya R, Ramirez-Estudillo JA, Sjoholm-gomez de Liano C, et al. Inverted ILM flap, free ILM flap and conventional ILM peeling for large macular holes. Int $J$ Retina Vitreous. 2018;4:1-9. doi:10.1186/s40942-018-0111-5

10. Yamashita T, Sakamoto T, Terasaki H, et al. Best surgical technique and outcomes for large macular holes: retrospective multicentre study in Japan. Acta Ophthalmol. 2018;96:e904-e910. doi:10.1111/ aos. 13795

11. Michaelewska Z, Michalewski J, Adelman RA, et al. Inverted internal limiting membrane flap technique for large macular hole. Ophthalmology. 2010;117:2018-2025. doi:10.1016/j.ophtha.20 10.02.011

12. Rizzo S, Tartaro R, Barca F, et al. Internal limiting membrane peeling versus inverted flap technique for treatment of full-thickness macular hole: a comparative study in a large series of patients. Retina. 2018;38(Suppl 1):S73-S78. doi:10.1097/IAE.0000000000001985

13. Duker JS, Kaiser PK, Binder S, et al. The International Vitreomacular Traction Study Group classification of vitreomacular adhesion, traction and macular hole. Ophthalmology. 2013;120:2611-2619. doi:10.1016/j.ophtha.2013.07.042

14. Liu P, Sun Y, Dong C, et al. A new method to predict anatomical outcome after idiopathic macular hole surgery. Arch Clin Exp Ophthalmol. 2016;254:683-688. doi:10.1007/s00417-015-3116-x

15. Michalewska Z, Michalewski J, Cisiecki S, et al. Correlation between foveal structure and visual outcome following macular hole surgery: a spectral optical coherence tomography study. Graefes Arch Clin Exp Ophthalmol. 2008;246:823-830. doi:10.1007/s00417-007-0764-5

16. Ip MS, Baker BJ, Duker JS, et al. Anatomical outcomes of surgery for idiopathic macular hole as determined by optical coherence tomography. Arch Ophthalmol. 2002;120:29-35. doi:10.1001/ archopht.120.1.29

17. Susini A, Gastaud P. Macular holes that should not be operated. $J \mathrm{Fr}$ Ophthalmol. 2008;31:214-220. doi:10.1016/S0181-5512(08)70359-0

18. Bae K, Kang SW, Kim JH, et al. Extent of internal limiting membrane peeling and its impact on macular hole surgery outcomes: a randomized trial. Am $J$ Ophthalmol. 2016;169:179-188. doi:10.1016/j.ajo.2016.06.041

19. Shukla SY, Afshar Arm Kierman DF, Hariprasad SM. Outcomes of chronic macular hole surgical repair. Indian $J$ Ophthalmol. 2014;62:795-798. doi:10.4103/0301-4738.138302

20. Gu C, Qiu Q. Inverted internal limiting membrane flap technique for large macular holes: a systematic review and single-arm meta-analysis. Graefes Arch Clin Exp Ophthalmol. 2018;256:1041-1049. doi:10.1007/s00417-018-3956-2

21. Marques RE, Sousa DC, Leal I, et al. Complete ILM peeling versus inverted flap technique for macular hole surgery: a meta-analysis. Ophthalmic Surg Lasers Imaging Retina. 2020;51:187-A2. doi:10.3928/23258160-20200228-08

22. Ishida M, Ichikawa $\mathrm{Y}$, Higashida $\mathrm{R}$, et al. Retinal displacement toward optic disc after internal limiting membrane peeling for idiopathic macular hole. Am J Ophthalmol. 2014;157:971-977. doi:10.1016/j.ajo.2014.01.026

23. Steel DH, Dinah C, White K, et al. The relationship between a dissociated optic nerve fiber layer appearance after macular hole surgery and Muller cell debris on peeled internal limiting membrane. Acta Ophthalmol. 2017;95:153-157. doi:10.1111/aos.13195

24. Cicinelli MV, Marchese A, Bandello F, et al. Inner retinal layer and outer retinal layer findings after macular hole surgery assessed by means of optical coherence tomography. $J$ Ophthalmol. 2019;2019:3821479. doi:10.1155/2019/3821479

25. Almony A, Nudleman E, Shah GK, et al. Techniques, rationale, and outcomes of internal limiting membrane peeling. Retina. 2012;32:877-891. doi:10.1097/IAE.0b013e318227ab39

26. Chatziralli IP, Theodossiadis PG, Steel DHW. Internal limiting membrane peeling in macular hole surgery; Why, When, and How? Retina. 2018;38:870-882. doi:10.1097/IAE.0000000000001959 
27. Modi A, Giridhar A, Gopalakrishnan M. Comparative analysis of outcomes with variable diameter internal limiting membrane peeling in surgery for idiopathic macular hole repair. Retina. 2017;37:265-273. doi:10.1097/IAE.0000000000001123

28. Yao Y, Qu J, Dong C, et al. The impact of extent of internal limiting membrane peeling on anatomical outcomes of macular hole surgery: results of a 54-week randomized clinical trial. Acta Ophthalmol. 2019;97:303-312. doi:10.1111/aos.13853

29. Hecht I, Mimouni M, Blumenthal EZ, et al. Sulfur hexafluoride $\left(\mathrm{SF}_{6}\right)$ versus perfluoropropane $\left(\mathrm{C}_{3} \mathrm{~F}_{8}\right)$ in the intraoperative management of macular holes: a systematic review and meta-analysis. J Ophthalmol. 2019;2019:1820850. doi:10.1155/2019/1820850

30. Inoue M, Watanabe Y, Arakawa A, et al. Spectral-domain optical coherence tomography images of inner/outer segment junctions and macular hole surgery outcomes. Graefes Arch Clin Exp Ophthalmol. 2009;247:325-330. doi:10.1007/s00417-008-0999-9

31. Itoh $\mathrm{Y}$, Inoue M, Rii $\mathrm{T}$, et al. Asymmetrical recovery of cone outer segment tips line and foveal displacement after successful macular hole surgery. Invest Ophthalmol Vis Sci. 2014;55:3003-3011. doi:10.1167/iovs.14-13973
32. Kim SH, Kim HK, Yang JY, et al. Visual recovery after macular hole surgery and related prognostic factors. Korean $J$ Ophthalmol. 2018;32:140-146. doi:10.3341/kjo.2017.0085

33. Kusuhara S, Negi A. Predicting visual outcome following surgery for idiopathic macular holes. Ophthalmologica. 2014;231:125-132. doi: $10.1159 / 000355492$

34. Ch'ng SW, Patton N, Ahmed M, et al. The Manchester large macular hole study: is it time to reclassify large macular holes? Am J Ophthalmol. 2018;195:36-42. doi:10.1016/j.ajo.2018.07.027

35. Ehlers JP, Uchida A, Srivastava SK, et al. Predictive model for macular hole closure speed: insights from intraoperative optical coherence tomography. Transl Vis Sci Technol. 2019;8:18. doi:10.1167/tvst.8.1.18
Clinical Ophthalmology

\section{Publish your work in this journal}

Clinical Ophthalmology is an international, peer-reviewed journal covering all subspecialties within ophthalmology. Key topics include: Optometry; Visual science; Pharmacology and drug therapy in eye diseases; Basic Sciences; Primary and Secondary eye care; Patient Safety and Quality of Care Improvements. This journal is indexed on PubMed

Submit your manuscript here: https://www.dovepress.com/clinical-ophthalmology-journal
Dovepress

Central and CAS, and is the official journal of The Society of Clinical Ophthalmology (SCO). The manuscript management system is completely online and includes a very quick and fair peer-review system, which is all easy to use. Visit http://www.dovepress.com/ testimonials.php to read real quotes from published authors. 